\title{
Femtosecond study of self-trapped vibrational excitons in crystalline acetanilide
}

\author{
Edler, J.; Hamm, Peter; Scott, Alwyn C.
}

Published in:

Physical Review Letters

Link to article, DOI:

10.1103/PhysRevLett.88.067403

Publication date:

2002

Document Version

Publisher's PDF, also known as Version of record

Link back to DTU Orbit

Citation (APA):

Edler, J., Hamm, P., \& Scott, A. C. (2002). Femtosecond study of self-trapped vibrational excitons in crystalline acetanilide. Physical Review Letters, 88(6), 067403. https://doi.org/10.1103/PhysRevLett.88.067403

\section{General rights}

Copyright and moral rights for the publications made accessible in the public portal are retained by the authors and/or other copyright owners and it is a condition of accessing publications that users recognise and abide by the legal requirements associated with these rights.

- Users may download and print one copy of any publication from the public portal for the purpose of private study or research.

- You may not further distribute the material or use it for any profit-making activity or commercial gain

- You may freely distribute the URL identifying the publication in the public portal

If you believe that this document breaches copyright please contact us providing details, and we will remove access to the work immediately and investigate your claim. 


\title{
Femtosecond Study of Self-Trapped Vibrational Excitons in Crystalline Acetanilide
}

\author{
J. Edler and P. Hamm \\ Max Born Institut für Nichtlineare Optik und Kurzzeitspektroskopie, Max Bornstrasse 2a, 12489 Berlin, Germany \\ A. C. Scott \\ Department of Mathematics, University of Arizona, Tucson, Arizona 85721 \\ and Department of Informatics and Mathematical Modelling, Technical University of Denmark, DK-2800 Lyngby, Denmark
}

(Received 21 June 2001; published 25 January 2002)

\begin{abstract}
Femtosecond IR spectroscopy of delocalized NH excitations of crystalline acetanilide confirms that self-trapping in hydrogen-bonded peptide units exists and does stabilize the excitation. Two phonons with frequencies of 48 and $76 \mathrm{~cm}^{-1}$ are identified as the major degrees of freedom that mediate self-trapping. After selective excitation of the free exciton, self-trapping occurs within a few $100 \mathrm{fs}$. Excitation of the self-trapped states disappears from the spectral window of this investigation on a 1 ps time scale, followed by a slow ground state recovery of the hot ground state within $18 \mathrm{ps}$.
\end{abstract}

DOI: 10.1103/PhysRevLett.88.067403

PACS numbers: 78.47.+p, 63.20.-e, 71.38.Ht

Nonlinear localization of energy in systems with translational invariance (sometimes called "self-trapping" or "autolocalization") is of growing interest, having been observed or speculated upon in a variety of contexts [1-10]. In addition to a general theoretical interest and specific technical applications (e.g., as a novel means to generate and detect far-IR electromagnetic radiation), it was suggested many years ago [11] that self-localized vibrational states might localize and stabilize small energy quanta, and, hence, might play an important role in one of the most remarkable - yet not well understood - properties of proteins and enzymes, namely, their capability of storing and transporting energy in an efficient manner.

Crystalline acetanilide (ACN) has been widely used as a simple model system to study this phenomenon $[2,3,5,12-17]$. It consists of quasi-one-dimensional chains of hydrogen-bonded peptide groups with structural properties that are comparable to those of an $\alpha$ helix, one of the most common structure motifs of proteins. In these hydrogen-bonded molecular crystals, two coupling mechanisms have to be discussed to obtain self-trapping [12]: (i) The so-called amide modes, i.e., the normal modes of the peptide units (- $\mathrm{CO}-\mathrm{NH}-)$, are known to be coupled by dipole-dipole interaction and to form delocalized states. In analogy to electronic molecular excitons [18], these states are commonly called "vibrational excitons" (even though, strictly speaking, they are "internal phonons"). (ii) These vibrational excitons are coupled to lattice phonons (i.e., external phonons) by nonlinear interaction, which is mediated through the hydrogen bonds stabilizing the crystal structure [19]. Excitation of an exciton, hence, leads to a deformation of the lattice, thereby localizing the state.

It was recognized quite some time ago that the $\mathrm{C}=\mathrm{O}$ stretching and $\mathrm{NH}$ stretching band of crystalline ACN exhibit "anomalies" (see Refs. [2,13,20] and Ref. [12] for a recent review article). In particular, the $\mathrm{NH}$ stretching mode, which is the focus of the present Letter, exhibits a main peak at $3295 \mathrm{~cm}^{-1}$ accompanied by an almostregular sequence of nine satellite peaks towards lower frequencies with an average spacing of about $55 \mathrm{~cm}^{-1}$ (see Fig. 1a and Refs. $[14,16,20])$. It has been proposed that the peculiarities in the vibrational spectra can be explained by self-trapping theory [3,12-15]. The intriguing feature of this theory is that all parameters can be determined by independent experiments [14]. Conventional models explaining the "anomalous" spectra of ACN, such as Fermi resonances and structural defects [5,16,17], have tentatively been discarded [12,13]. Interestingly, many anharmonic features of the spectra are not observed in amorphous ACN $[2,20]$. The small Davidov splitting of the main NH peak at $3295 \mathrm{~cm}^{-1}$ shows that this band is indeed a delocalized state [20]. We shall refer to this peak as the free-exciton peak. However, since nearest neighbor coupling is much smaller than the binding energy of the self-trapped states, it is commonly neglected in theoretical
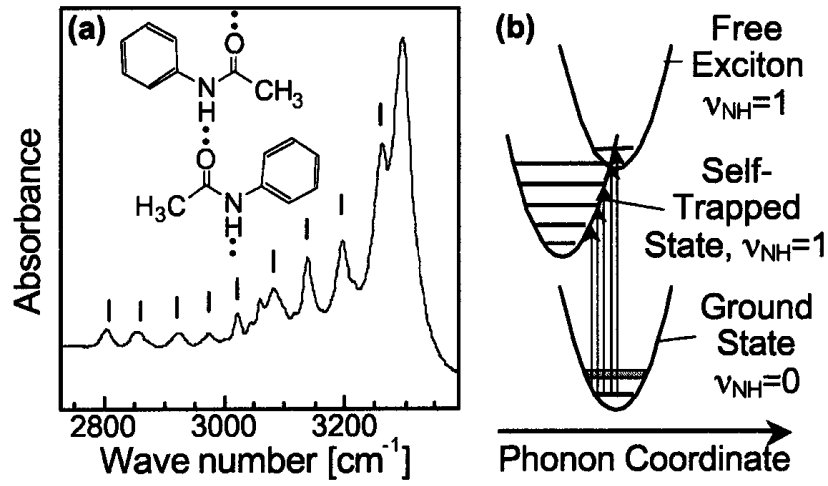

FIG. 1. (a) Absorption spectrum of the $\mathrm{NH}$ band of crystalline ACN (see also $[14,16,20]$ ), showing the main peak at $3295 \mathrm{~cm}^{-1}$ (i.e., the free-exciton peak) and a sequence of nine almost equidistant satellite peaks (i.e., the self-trapped states). The inset schematically depicts the one-dimensional chain of hydrogen bonded ACN molecules. (b) The proposed scheme of potential surfaces with the allowed transitions depicted. 
treatments $[12,14,15]$. This leads to the problem of the "displaced oscillator" for each lattice site, which has been used before to discuss the linear [19] and nonlinear [21] spectroscopy of hydrogen bonds of isolated molecules. The problem is simplified significantly by introducing an adiabatic, "Born Oppenheimer"-like, separation of time scales of phonon and $\mathrm{NH}$ stretching degrees of freedom, leading to potential energy surfaces as functions of phonon coordinates for each excitation level of the high-frequency $\mathrm{NH}$ stretching mode. Nonlinear interaction between phonon and $\mathrm{NH}$ excitation is responsible for a displacement of the potential energy surfaces of the so-called self-trapped states, for which a "Franck-Condon" progression is obtained consisting of one $\mathrm{NH}$ excitation plus several quanta of phonon excitations (i.e., the regular sequence of nine satellite peaks). Exciton coupling, which is treated perturbatively, is responsible for the sudden cutoff of Franck-Condon transitions above the free-exciton peak at $3295 \mathrm{~cm}^{-1}$ [14]. This is a result of the instability of the self-trapped states once their energy exceeds that of the free, delocalized exciton. Hence, a scheme with three potential surfaces is proposed (Fig. 1b: (i) The NH ground state with the phonon ground state and a band of delocalized phonons; (ii) the delocalized free-exciton potential surface, which is not displaced with respect to the ground state potential surface and, hence, does not involve phonon excitation; and (iii) the self-trapped states, for which both the $\mathrm{NH}$ excitation and the phonons are localized.

To test these ideas, femtosecond IR pump-probe experiments were performed with pulses centered at about $3200 \mathrm{~cm}^{-1}$, pulse duration $130 \mathrm{fs}$, bandwidth $200 \mathrm{~cm}^{-1}$ (FWHM), and pulse energy 1-2 $\mu \mathrm{J}$ [22]. A small fraction of the infrared pulses was split off to obtain broad band probe pulses which were spectrally dispersed after interaction with the sample and detected with a 31 channel $\mathrm{HgCdTe}$ detector array $\left(8 \mathrm{~cm}^{-1}\right.$ resolution), enabling us to measure transient spectra with a dynamic resolution of $\approx 5 \times 10^{-6}$. The remainder was used as a pump pulse, which was spectrally filtered for some of the experiments using an adjustable Fabry-Perot filter (bandwidth $30 \mathrm{~cm}^{-1}$, pump pulse duration $\sim 250 \mathrm{fs}$ FHWM). Zone refined ACN was obtained from Aldrich (purity 99.95\%). Monocrystalline ACN (thickness $\approx 1 \mu \mathrm{m}$ along the $c$ axis) was prepared by cooling a thin layer of melted ACN between two $\mathrm{CaF}_{2}$ windows. Pump and probe pulses were polarized parallel to the NH bond (the $b$ axis). All experiments were performed at room temperature.

In a first experiment, short and broad band pump pulses were used to impulsively excite a section of the absorption spectrum which includes the $\mathrm{NH}$ free-exciton peak and the first three satellite peaks. The transient absorbance change for probe frequencies corresponding to these four absorption peaks is shown in the inset of Fig. 2. At delay time zero, this signal is strongly influenced by cross phase modulation, caused by Kerr nonlinearities of the crystal and the $\mathrm{CaF}_{2}$ sample cell. Hence, we restrict the discus-

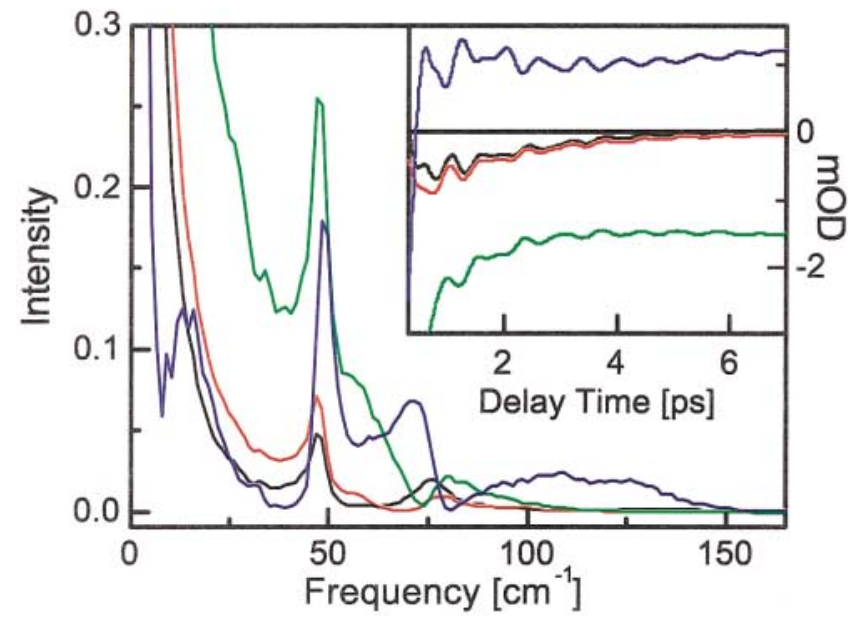

FIG. 2 (color). Fourier transform spectra of the coherent response ACN after impulsive excitation. The inset shows the corresponding signals in the time domain. The four signals correspond to the absorption peaks in the linear spectrum: $3295 \mathrm{~cm}^{-1}$ (blue line), $3260 \mathrm{~cm}^{-1}$ (green line), $3195 \mathrm{~cm}^{-1}$ (red line), and $3137 \mathrm{~cm}^{-1}$ (black line).

sion of the data to delay times $>300$ fs only, when temporal overlap of pump and probe pulse is essentially over. The striking feature is pronounced oscillations that are very reproducible and persist up to about 8 ps. The Fourier transform spectra of the transients (Fig. 2), which are rich in structure and highly reproducible, clearly contain a major frequency component at $48 \mathrm{~cm}^{-1}$ and a weaker one at about $76 \mathrm{~cm}^{-1}$.

There are two possibilities for the ultrashort pump pulse to interact with the system [23]: both field interactions going "up-up," yielding an excited state population and an excited state wave packet, and both field interactions going "up-down," yielding no population of the excited state but a ground state wave packet. Both processes are expected to occur simultaneously with a probability given by the Franck-Condon factors of the individual transitions. The beating frequencies of the two dominant oscillating features at 48 and $76 \mathrm{~cm}^{-1}$ help to assign the wave packets. On the one hand, the splittings between the sublevels of the $\mathrm{NH}$ absorption band do not resemble these frequencies (see arrows in Fig. 3a), which would be what is expected for an excited state wave packet. To be more precise, at a probe frequency resonant with a particular sublevel, one expects to observe a beating frequency which reflects the energy splitting between this state and adjacent states which share the common ground state [24]. When taking the free-exciton peak as an example $\left(3295 \mathrm{~cm}^{-1}\right)$, this could be a beating frequency of $35 \mathrm{~cm}^{-1}, 100 \mathrm{~cm}^{-1}$ (i.e., $35 \mathrm{~cm}^{-1}+65 \mathrm{~cm}^{-1}$ ), etc. The sharp bands at 48 and $76 \mathrm{~cm}^{-1}$ clearly do not fit into this scheme. On the other hand, two bands can be identified in the nonresonant electronic Raman spectrum of ACN [13], whose frequencies match perfectly with the beating frequencies observed in our pump-probe experiment. We therefore conclude that the beating structure represents a long-lived 

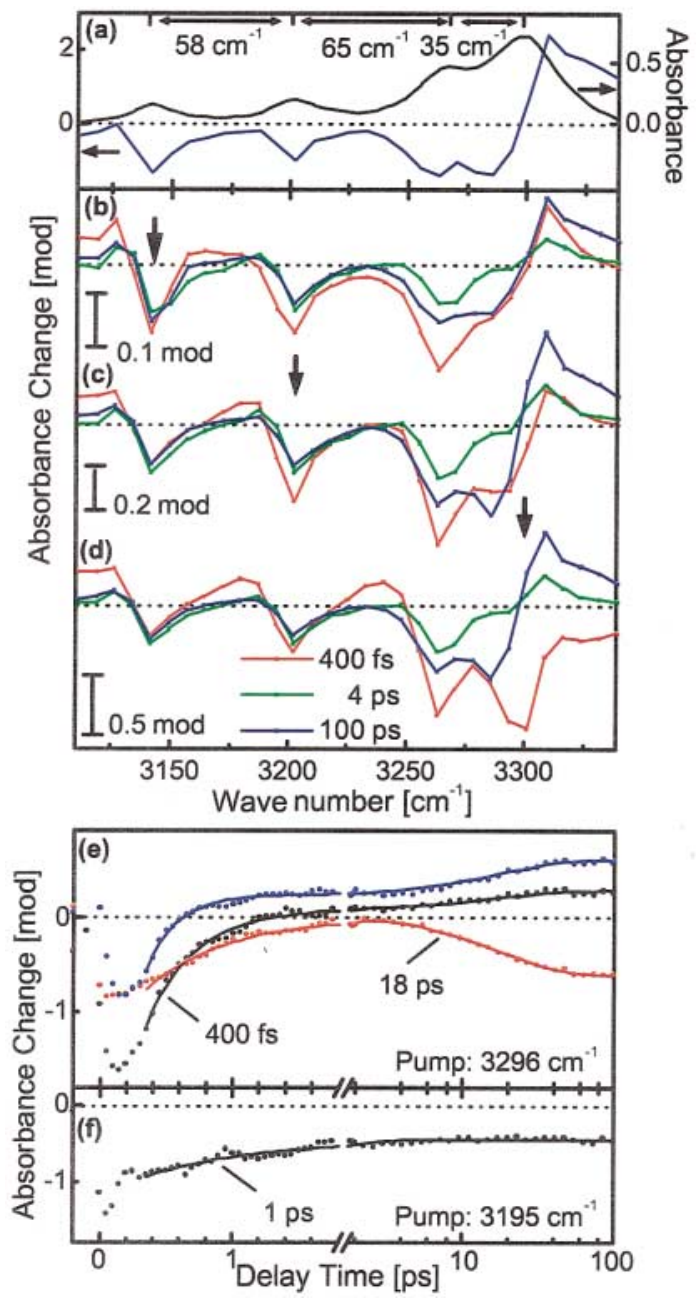

FIG. 3 (color). (a) Part of the NH band of crystalline ACN (black line) together with the temperature induced absorbance change upon heating of $3{ }^{\circ} \mathrm{C}$ (blue line). (b)-(d) Response of the sample upon selective excitation of the self-trapped states at $3137 \mathrm{~cm}^{-1}$ (b) and at $3195 \mathrm{~cm}^{-1}$ (c), and the free-exciton peak (d) for delay times of $400 \mathrm{fs}$ (red lines), $4 \mathrm{ps}$ (green lines), and $100 \mathrm{ps}$ (blue lines), respectively. The position of the narrow band pump pulse (spectral width $30 \mathrm{~cm}^{-1}$ FWHM) is indicated by the arrow. (e) Temporal evolution of the transient signal after selective excitation of the free-exciton peak for probe frequencies at the red wing $\left(3280 \mathrm{~cm}^{-1}\right.$, red line), center (3295 $\mathrm{cm}^{-1}$, black line), and blue wing $3303 \mathrm{~cm}^{-1}$, blue line) of the band, respectively. (f) Temporal evolution of the self-trapped state at $3195 \mathrm{~cm}^{-1}$ after selectively pumping at the same frequency position.

ground state wave packet generated through a "stimulated impulsive Raman effect." In contrast to the congested electronic Raman spectrum of Ref. [13], this is a "resonant Raman effect" enhanced by the NH absorption band as a consequence of anharmonic coupling to some of the phonons [21]. Hence, selection rules apply, reducing the number of "resonant Raman active" phonons. These are the phonons which mediate self-trapping. In order to be resonant Raman active, the lattice deformation has to lower the total hydrogen bond energy by an overall shortening of the hydrogen bond distances. This would be, for example, a torsion of the tilted ACN molecules, straightening the hydrogen bond chain. Only two such phonons with frequencies of 48 and $76 \mathrm{~cm}^{-1}$ exist within the frequency window determined by the time resolution of our experiment $\left(0-250 \mathrm{~cm}^{-1}\right)$.

In addition, weaker broad features are observed in the Fourier transform spectra, which are assigned to shortlived excited state wave packets. These are a band around $100 \mathrm{~cm}^{-1}$ in the $3295 \mathrm{~cm}^{-1}$ data (Fig. 2, blue line) and a broad shoulder at about $65 \mathrm{~cm}^{-1}$ in the $3260 \mathrm{~cm}^{-1}$ data (Fig. 2, green line). In both cases, their frequencies fit well into the scheme for excited state motion described above. From the width of these bands $\left(\approx 30 \mathrm{~cm}^{-1}\right)$, an excited state lifetime of about $300 \mathrm{fs}$ is estimated. Since ground and excited state wave packets have altered frequencies, both potential surfaces must be different.

In a second set of experiments, narrow band, tunable pump pulses (spectral width $30 \mathrm{~cm}^{-1}$, pulse duration $250 \mathrm{fs}$ FWHM) were used to selectively excite individual sublevels. Figures $3 b-3 d$ show the response of the sample after excitation of self-trapped states (at 3137 and $3195 \mathrm{~cm}^{-1}$, respectively) and the free-exciton peak for delay times of $0.4,4$, and $100 \mathrm{ps}$. The system equilibrates rapidly on a ps time scale, resulting in spectra which do not depend on pump frequency after a delay time of 4 ps (Figs. 3b-3d, green lines). However, on the subpicosecond time scale, the free-exciton and the lower lying self-trapped states behave distinctly different (Figs. 3b-3d, red lines). When exciting the free exciton, a strong bleach and stimulated emission signal is observed, which recovers on a $400 \pm 100 \mathrm{fs}$ time scale, representing the free-exciton lifetime (Fig. 3e, black line). Simultaneously, population is transferred into lower lying self-trapped states, giving rise to negative peaks that are larger in the $400 \mathrm{fs}$ spectrum than in the 4 ps spectrum. This can be seen after subtracting a broad positive background, reflecting anharmonically redshifted $\nu=1 \rightarrow \nu=2$ excited state absorption of the free exciton. On the other hand, when pumping one of the self-trapped states directly (Figs. $3 b$ and $3 c$ ), the population in between all self-trapped states equilibrates essentially instantaneously, yielding an additional negative signal of all of these states in the $400 \mathrm{fs}$ spectrum, which is almost independent on which state has been pumped (compare Figs. 3b and 3c). However, the free-exciton peak is not back-populated. This is a direct observation of ultrafast self-trapping: Excitation of the free-exciton leads to an irreversible population of self-trapped states. Since a back-transfer would require restoration of phonon coordinates, it does not occur even though it would be possible energetically within $k T$.

The difference spectra measured $100 \mathrm{ps}$ after excitation (Figs. 3b-3d, blue lines) and that induced by a stationary temperature increase (Fig. 3a, blue line) are essentially identical. Hence, we conclude that the 100 ps merely mirrors the temperature jump induced by the intense pump pulse, and the bleach of the 3137, 3195, and $3260 \mathrm{~cm}^{-1}$ band reflects a weakening of the self-trapping 
mechanism $[14,15]$, rather than excitation of these states. Again, the free-exciton peak responds in a distinctly different way to such a temperature jump. Rather than a bleach, a shift towards a higher frequency is found which occurs on a relatively slow $18 \pm 3$ ps time scale [i.e., a negative signal at the red side and a positive signal at the blue side of the band (see Fig. $3 \mathrm{e}$ blue and red line)]. Such a blueshift is a well-known phenomenon for hydrogen-bonded systems [25]: As a consequence of the anharmonicity of the hydrogen bond potential, the average hydrogen bond distance increases with excitation of those modes, which modulate the hydrogen bond distance. Since the frequency of the NH vibration depends on the hydrogen bond distance [19], the position of the free-exciton peak is a measure of the excitation of these modes, or, after thermalization, a measure of temperature, respectively. Furthermore, as these modes modulate the $\mathrm{NH}$ absorption frequency, these must be the resonance Raman active phonons. Owing to the large displacement of the potential surface along these coordinates corresponding to a binding energy of $\approx 500 \mathrm{~cm}^{-1}$, these phonons are certainly excited directly upon relaxation of the system into the ground state, rather than delayed through indirect channels. Hence, combining both arguments, we conclude that the appearance of the blueshift within $18 \pm 3 \mathrm{ps}$ mirrors ground state recovery.

Interestingly, the lifetime of the initially excited states is much shorter than the $18 \pm 3$ ps ground state recovery. This is directly evident for the free-exciton peak with a bleach recovery time of $400 \pm 100$ fs (Fig. 3e, black line). The self-trapped states equilibrate very quickly on a time scale which is in the same order as the time resolution of this experiment. These fast relaxation and equilibration times are in agreement with the absence and/or short lifetime of excited state wave packets. Besides, they are still consistent with the bandwidth of the sublevels [i.e., $\geq(1 / 2 \pi) \Delta \omega]$. The initially excited states relax on a $1 \pm 0.3$ ps time scale (Fig. 3f) into states that are either outside of our frequency window or spectroscopically dark, and return back to the ground state with a delay of $18 \pm 3$ ps. At this point, we can only speculate on the nature of these states. However, as equilibration between the various self-trapped states is extremely fast, one possibility is relaxation towards the bottom of the self-trapped potential surface.

In conclusion, we have reported direct observation of self-trapping of NH-vibrational excitons in crystalline $\mathrm{ACN}$. It is relevant to note that ground state recovery is about 20 times faster $(\approx 800 \pm 200 \mathrm{fs}$, data not shown $)$ for isolated ACN molecules dissolved in acetone, a solvent which forms hydrogen bonds to the $\mathrm{NH}$ group of ACN with approximately the same strength as those in the crystal. Hence, it is the crystalline structure which enables self-trapping, and which is indeed capable of stabilizing the excitation, an important condition for biological relevance. Even though the lifetime of the excitation (20 ps) is not quite as long as initially anticipated by Davidov [11], it appears sufficient to transport energy over significant distances of a few tens of $\AA$. Moreover, these experiments make evident that the large nonlinearity of the hydrogen bonds, which stabilize regular protein structures, gives rise to a rich variety of collective excitations that have yet to be explored.

This work was funded by the Deutsche Forschungsgemeinschaft (SF450). We thank T. Elsaesser for continuous support of the project.

[1] F. Tappert and C. M. Varma, Phys. Rev. Lett. 25, 1108 (1970).

[2] G. Careri, U. Buontempo, F. Carta, E. Gratton, and A.C. Scott, Phys. Rev. Lett. 83, 304 (1983).

[3] D. M. Alexander, Phys. Rev. Lett. 54, 138 (1985).

[4] St. Pnevmatikos, Phys. Rev. Lett. 60, 1534 (1988).

[5] W. Fann, L. Rothberg, M. Roberson, S. Benson, J. Madey, S. Etemad, and R. Austin, Phys. Rev. Lett. 64, 607 (1990).

[6] R. Bruinsma, K. Maki, and J. Wheatley, Phys. Rev. Lett. 57, 1773 (1986).

[7] A. J. Sievers and S. Takeno, Phys. Rev. Lett. 61, 970 (1988).

[8] D. Chen, S. Aubry, and G. P. Tsironis, Phys. Rev. Lett. 77, 4776 (1996).

[9] D. Abramov, P. Caputo, G. Filatrella, M. V. Fistul, G. Yu. Logvenov, and A. V. Ustinov, Phys. Rev. Lett. 83, 5354 (1999).

[10] A. Xie, L. van der Meer, and R. H. Austin, Phys. Rev. Lett. 23, 5435 (2000).

[11] A. S. Davidov, J. Theor. Biol. 66, 379 (1977).

[12] A. C. Scott, Phys. Rep. 217, 1 (1992).

[13] G. Careri, U. Buontempo, F. Galluzzi, A. C. Scott, E. Gratton, and E. Shyamsunder, Phys. Rev. B 30, 4689 (1984).

[14] D. M. Alexander and J. A. Krumhansl, Phys. Rev. B 33, 7172 (1986).

[15] A. C. Scott, I. J. Bigio, and C. T. Johnston, Phys. Rev. B 39, 12883 (1989).

[16] G. C. Blanchet and C. R. Fincher, Phys. Rev. Lett. 54, 1310 (1985).

[17] C. T. Johnston and B. I. Swanson, Chem. Phys. Lett. 114, 547 (1985).

[18] A.S. Davidov, Theory of Molecular Excitons (Plenum, New York, 1971).

[19] O. Henri-Rousseau and P. Blaise, Adv. Chem. Phys. 103, 1 (1998).

[20] N. B. Abbott and A. Elliott, Proc. R. Soc. London A 234, 247 (1956).

[21] D. Madsen, J. Stenger, J. Dreyer, E. T. J. Nibbering, P. Hamm, and T. Elsaesser, Chem. Phys. Lett. 341, 56 (2001).

[22] P. Hamm, R. A. Kaindl, and J. Stenger, Opt. Lett. 25, 1798 (2000).

[23] S. Mukamel, Principles of Nonlinear Optical Spectroscopy (Oxford University Press, Oxford, 1995).

[24] P. Hamm, M. Lim, W. F. DeGrado, and R. M. Hochstrasser, J. Chem. Phys. 112, 1907 (2000).

[25] H. K. Nienhuys, S. Woutersen, R. A. van Santen, and H. J. Bakker, J. Chem. Phys. 111, 1494 (1999). 